\title{
Some Approaches to Mass Reduction of the Gasdynamic Mirror Fusion Rocket
}

\author{
T. Kammash \\ Department of Nuclear Engineering and Radiological Sciences \\ The University of Michigan \\ Ann Arbor, MI 48109 USA
}

\begin{abstract}
Previous studies have shown that the gasdynamic mirror (GDM) fusion propulsion system is capable of producing specific impulses in excess of $10^{5}$ seconds and thrusts in the tens of kilonewtons. These propulsive capabilities arise from the ability of this magnetic fusion system to confine a hot plasma long enough to produce fusion energy while allowing a certain fraction of its charged particle population to escape through one end (a magnetic nozzle) to generate thrust. Earlier investigations have revealed that the optimum performance arise from the use of large mirror ratios which require large magnets and result in very massive vehicles. Major contributors to the large mass, in addition to the magnetic fields, are the large radiators required to dispose of waste heat. In this paper we address the question of mass reduction by investigating the role of magnetic field reversal near the mirror region, on the one hand, and the utilization of the liquid droplet radiator design on the other. We find that significant reduction in the mass of the vehicle can be achieved, and a reduction in cost and travel time are the direct beneficiaries of this reduction.
\end{abstract}

\section{Introduction \\ The gasdynamic mirror fusion} propulsion concept shown in Fig. 1 makes use of a magnetic mirror confinement system in which the plasma has a density and temperature that make the ion collision

Copyright $(\mathcal{O} 1998$ by the American Institute of Aeronautics and Astronautics, Inc. All rights reserved. mean free path much shorter than the length of the device. Under these conditions the plasma behaves like a continuous medium a fluid, and its confinement is governed by gasdynamic laws. The escape of the plasma from the system is analogous to the flow of a gas into a vacuum from a vessel with a hole. The governing equations are the particle and energy conservation equations which when solved self-consistently yield the length of the device as a function of the plasma parameters. ${ }^{(1)}$ With the aid of a power flow diagram for a propulsion system that consists of the reactor, the injector, and a thermal and a direct converter, it has been shown $^{(2)}$ that the energy multiplication factor of the fusion reactor (the Q-value) has to be slightly larger than unity to accommodate the efficiencies of the various components. A preliminary design of such a propulsion system, given in Table 1 , reveals that for a deuterium-tritium (DT) fuel cycle, the rocket will be about $44 \mathrm{~m}$ long with a total vehicle mass of $422 \mathrm{mT}$. It will produce a specific impulse and thrust that allows a round trip mission to Mars to be accomplished in 169 days instead of several years. Of particular concern, however, is the mass of the magnets which can be very large especially at the mirror ends due to the large mirror ratio required for modest size devices. Also because of the relatively low efficiencies of the thermal converter $(30 \%-45 \%)$ a large amount of waste heat has to be disposed of by a large radiator whose mass is calculated on the basis of an ability to radiate $5 \mathrm{MW}$ of thermal power per metric ton of mass. The magnet mass shown in Table 1 assumes 


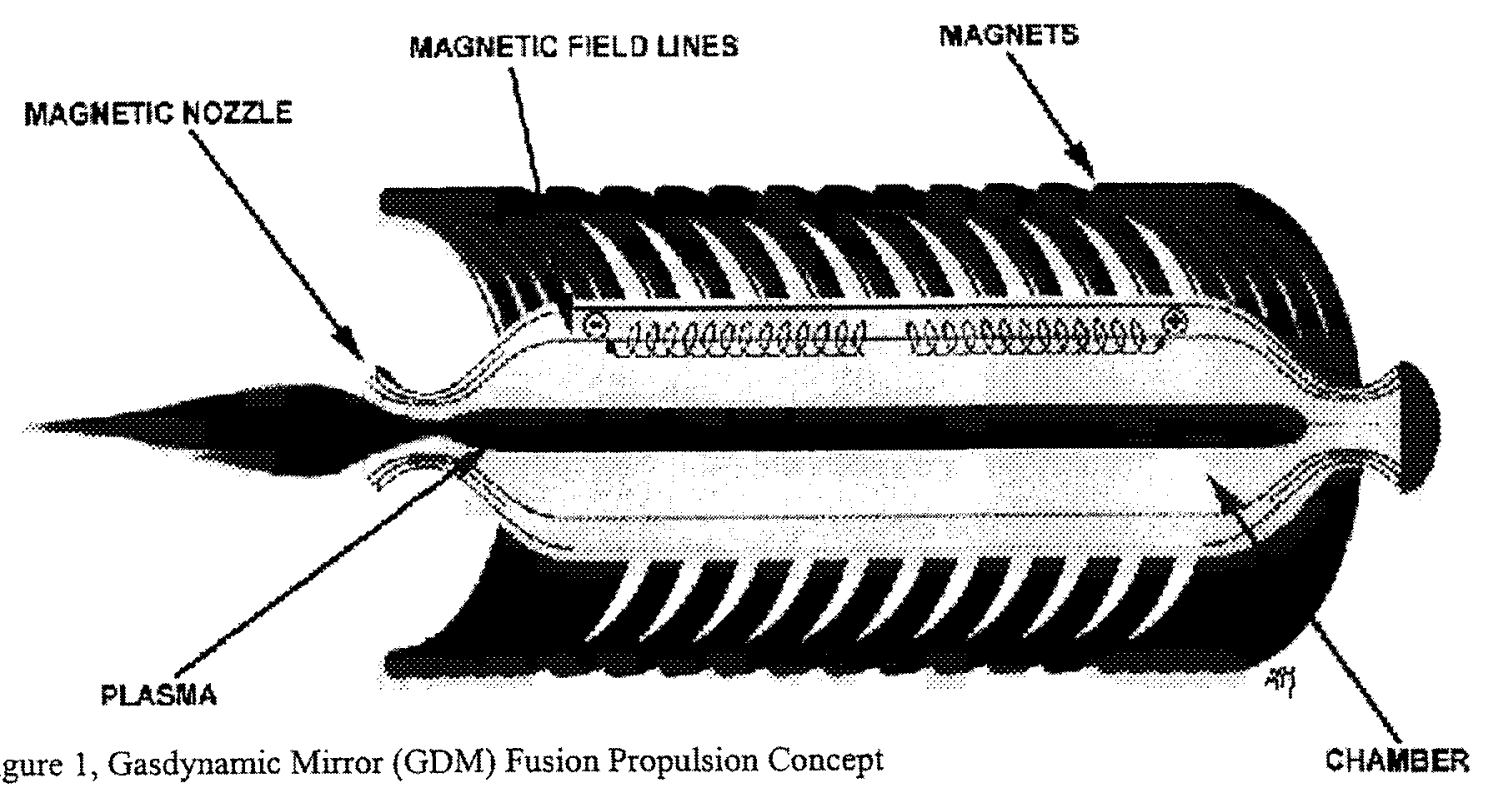

superconductivity magnets in with a current density of $250 \mathrm{MA} / \mathrm{m}^{2}$ that extend over the length of the machine including the mirror regions. This is clearly an approximation which results in a lower vehicle mass that must be addressed if it is to be viewed with some measure of realism. Magnetic field reversal has the potential of meeting this requirement. This and the liquid droplet radiator design which addresses the radiator problem will be examined in the next two sections.

\section{Field-Reversed GDM}

The concept of field reversal is illustrated in Fig. 2 where the plasma inside the separatrix sees closed magnetic field lines. The magnetic flux function that describes this configuration can be written as

$$
\Psi=\Psi_{0} \frac{r^{2}}{r_{0}^{4}}\left[2 \mathrm{r}_{0}^{2}-\mathrm{r}^{2}-\alpha^{2} z^{2}\right]
$$

where $\psi 0$ is the value of $\psi$ at the " 0 " where the magnetic field vanishes, and $\alpha^{2}$ is the ellipticity parameter. When $\alpha^{2}>1$ the configuration is "oblate," and it is "prolate" when $\alpha^{2}<1$ with the latter supporting less plasma pressure than the former. Outside the separatrix where $\psi=0$, the field lines are open. To generate this magnetic configuration an azimuthal current, $\mathrm{J}_{\theta}$, must be induced in the plasma, and recent experiments on "compact toroids" have shown ${ }^{(3)}$ that such a current can be induced by a rotating magnetic field (RMF). The first requirement on such a field is that the electrons of the plasma be magnetized while the ions are not. This condition is generally satisfied when the following is true:

$$
\begin{aligned}
& \omega_{\mathrm{ci}} \ll \omega_{\mathrm{RMF}} \ll \omega_{\mathrm{ce}} \\
& \omega_{\mathrm{ce}} \gg \gamma_{\mathrm{ei}}
\end{aligned}
$$

when $\omega_{\mathrm{ce}}$ and $\omega_{\mathrm{ci}}$ are the gyrofrequencies of the electron and ions in the rotating magnetic field respectively $\omega_{\mathrm{RMF}}$ the frequency of the rotating field, and $\gamma_{\mathrm{ei}}$ the electron-ion collision frequency. The second requirement on the rotating magnetic field is that it penetrates the plasma. Such penetration is represented by the "skin depth" given by 


\begin{tabular}{|l|c|}
\hline \multicolumn{1}{|c|}{ Parameter } & Value \\
\hline Plasma Density $\left(\mathrm{cm}^{-3}\right)$ & $1 \times 10^{16}$ \\
\hline Plasma Temperature (keV) & 10 \\
\hline Plasma Mirror Ratio & 100 \\
\hline Beta Value & .95 \\
\hline Central Magnetic Field (tesla) & 9.21 \\
\hline Plasma Radius (cm) & 5 \\
\hline Plasma Length (m) & 43.71 \\
\hline Confinement Time (sec) & $4.07 \times 10^{-3}$ \\
\hline Fusion Power (MW) & $2.73 \times 10^{3}$ \\
\hline Injection Power (MW) & $2.24 \times 10^{3}$ \\
\hline Bremsstrahlung Power (MW) & 58.17 \\
\hline Synchrotron Power (MW) & 18.94 \\
\hline Neutron Power (MW) & $2.19 \times 10^{3}$ \\
\hline Thrust Power (MW) & $1.35 \times 10^{3}$ \\
\hline Thrust (N) & $2.51 \times 10^{3}$ \\
\hline Reactor Mass & 55.50 \\
\hline Injector Mass & 45.40 \\
\hline Engine Mass (Reactor + Injector) & 100.90 \\
\hline Thermal Converter Mass (Mg) & 45.90 \\
\hline Direct Converter Mass (Mg) & 27.50 \\
\hline Radiator Mass (Mg) & 248.60 \\
\hline Total Vehicle Mass (Mg) & 422.90 \\
\hline Specific Impulse (sec) & $1.268 \times 10^{5}$ \\
\hline Specific Power (kW/kg) & 13.40 \\
\hline Mars Round Trip Time (days) & 169 \\
\hline
\end{tabular}

Table 1, GDM Parameters for DT fuel cycle.

$$
\delta=\left(\frac{z \eta}{\omega \mu_{0}}\right)^{1 / 2}
$$

where $\eta$ is the plasma resistivity, and $\mu_{0}$ the magnetic permeability. If we introduce an effective resistivity by

$$
\eta_{e f f}=\frac{\eta \omega_{c e}{ }^{2}}{2 \gamma_{e i}{ }^{2}}
$$

then an effective skin depth, $\delta_{\text {eff }}$ can be defined to have the form

$$
\delta_{e f f}=\left(\frac{\omega_{c e}}{\gamma_{e i}}\right) \delta
$$

This suggests that the rotating field has better penetration as the resistivity decreases and the plasma becomes more conductive.
This is expected to be the case in a GDM device operating as a propulsion system since the temperature will be sufficiently high to make the plasma nearly infinitely conducting.

For application to a GDM propulsion device we envisage creating the field reversed configuration in a region near the mirror ends of the device. By matching the magnetic field at the separatrix e.g. at point A, with about $90 \%$ of the mirror field of GDM i.e.

$$
\mathrm{BCA})=\frac{8 \Psi_{0}}{a^{2}} \hat{z}=0.9 B_{m}
$$

with $\underline{\underline{z}}$ denoting a unit sector in the $z$ direction, we can calculate $\Psi_{0}$ by specifying " $a$ " to be the width of the field-reversed region. Ninety percent reversal of the mirror field is necessitated by the need for a "hill" to prevent the field-reversed region from drifting outward. Knowing $\Psi_{0}$ allows us to calculate the azimuthal current required to achieve reversal in the desired region, and from the relation

$$
J_{o}=n e r_{0} \omega_{R M F}
$$

we can compute the frequency of the rotating field. In short it is possible in principle to create a GDM configuration where the central axial magnetic field is effectively all that is necessary to confine the plasma at large mirror ratios without having to actually construct very large magnets at the mirrors. The mass of the power source required to achieve this is negligibly small by comparison.

\section{Liquid Droplet Radiator}

As stated earlier, the radiator mass given in Table 1 is computed on the basis of $5 \mathrm{MW}$ per $\mathrm{mT}$. Considerable reduction in this mass can be achieved if a liquid droplet design is utilized. Such a design is illustrated in Fig. 3 where we note that it 


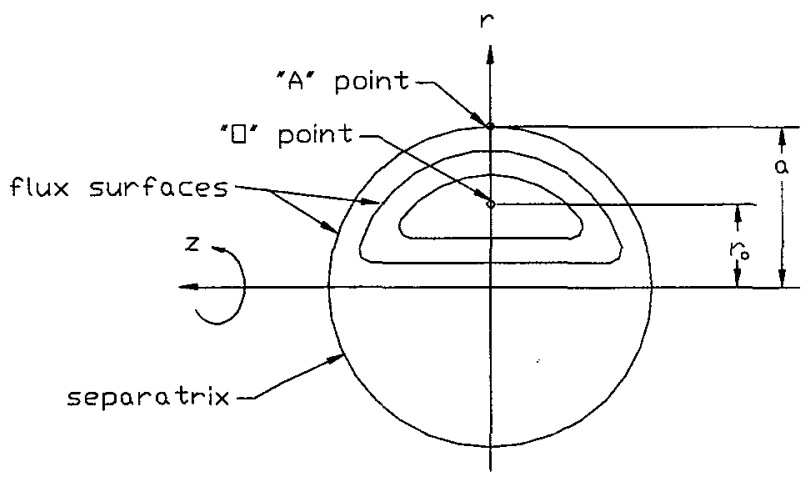

Figure 2, Field Reversed Concept

consists of a pair of parallel triangular droplet radiators. The droplet generator sprays the fluid towards a cylindrical collector where it is pressurized. The second radiator repeats the process returning the fluid to the heat exchanges thus neatly avoiding the extra mass of a return pipe. Usually four of these dual-sheet radiators are assumed to be spaced evenly around the vehicle circumference. The major advantages of such a radiator system include I) the radiating surface is invulnerable to puncture by micro-meteorite, II) it is light weight since very little fluid mass is required for a given radiating area and armoring against puncture is not required, III) the droplet generator and collector booms are easily deployed or stowed, IV) a long lifetime is assured by the choice of a low vapor pressure liquid.

The relevant parameters for the system are obtained as follows. Mass conservation at the droplet generator exit requires a mass flow rate of

$$
m_{f}^{0}=\frac{4}{3} \pi r^{3} p\left(\frac{u}{\lambda}\right)\left(\frac{s w}{d^{2}}\right)
$$

where $\lambda$ is the spacing between droplets in a stream, $d$ the spacing between separate streams, $s$ the droplet sheet thickness, $r$ the droplet radius, $\mathrm{p}$ the droplet density, w the radiator width, and $u$ the droplet speed. If we introduce the optical thickness, $\tau$, given by

$$
\tau=\pi r^{2} s /\left(d^{2} \lambda\right)
$$

then the mass flow rate can be written as

$$
m^{0}{ }_{f}=\frac{4}{3} r u p \tau A_{r} L
$$

where $L$ is the radiator length, and $A_{r}$ is its aspect ratio. Energy conservation at the droplet generator exit requires that the power $P_{r}$ be given by

$$
P_{r}=M_{f} C p \Delta T / L
$$

where $\mathrm{M}_{\mathrm{f}}$ is the total mass of the droplets in flight, $\Delta \mathrm{T}=\mathrm{T}_{0}-\mathrm{T}_{1}$ i.e. the droplet temperature change, and $\mathrm{Cp}$ the specific heat. The above equation yields for $M_{f}$ the value

$$
M_{f}=\frac{L}{u}\left(\frac{P_{r}}{C p \Delta T}\right)
$$

Since the power rejection is almost entirely radiative, ${ }^{(4)}$ energy conservation further requires that

$P_{r}=z \sigma \varepsilon A T e^{4}=m_{f} C p \Delta T$

where $\sigma$ is the Slefan-Boltzmann Constant, $\varepsilon$ the mean hemispherical emissivity of the droplet sheet, ${ }^{(5)} \mathrm{A}=\mathrm{wL} / \mathrm{z}$ the radiator projected area, and $\mathrm{T}_{\mathrm{e}}$ the effective mean radiator temperature given by

$$
\mathrm{T}_{\mathrm{e}}=\mathrm{T}_{0}\left(\frac{3}{f^{3}+f^{2}+f}\right)^{/ / 4}
$$

with $\mathrm{f}=\mathrm{T}_{0} / \mathrm{T}_{1}$. Combining Eqs. 12 and 9 we obtain for the radiator length the result 


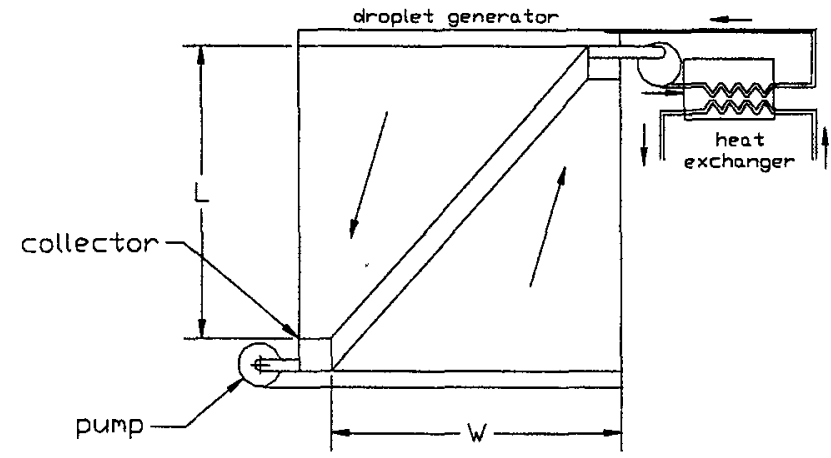

Figure 3, Liquid Droplet Radiator Design

$$
L=\frac{4}{3} \frac{u \tau r P \Delta T}{J \varepsilon T_{e}^{4}}
$$

To apply the above analysis to GDM we use the nominal values from a base line $100-\mathrm{MW}$ liquid droplet radiator ${ }^{(6)}$ where $\mathrm{T}_{\mathrm{o}}$ $=528^{\circ} \mathrm{K}, \mathrm{T}_{1}=460^{\circ} \mathrm{K}, \mathrm{f}=1.148$ and hence an effective temperature of $\mathrm{T}_{\mathrm{e}}=492^{\circ} \mathrm{K}$. A generator pressure of 8.5 atmospheres is assumed which leads to a "lithium" droplet ejection speed of $58 \mathrm{~m} / \mathrm{s}$. Assuming further a droplet radius of $\mathrm{r}=50 \mu \mathrm{m}$, an optical depth of $\tau=3$ and sheet emissivity of $\varepsilon=0.58$, the radiator length is found from Eq. 14 to be $\mathrm{L}=456 \mathrm{~m}$. For an aspect ratio of $A_{1}=0.4$ the mass flow rate is found to be $\mathrm{m}_{\mathrm{f}}=1.1 \times 10^{3} \mathrm{~kg} / \mathrm{s}$. Finally the in-flight mass for a single radiator which can dispose of GDM's waste heat can be computed by noting that the total radiated power is expressed by

$$
P_{r}=\eta_{m}\left(P_{B}+P_{S}+P_{n}\right)
$$

where $\eta_{\mathrm{th}}$ is the thermal converter efficiency, $P_{B}$ the bremsstrahlung power, $P_{s}$ the synchrotron radiation power and $P_{n}$ the neutron power. At $\eta_{\mathrm{th}}=0.45$ and the power shown in Table 1 we see that $\mathrm{P}_{\mathrm{r}}=1.243 \mathrm{MW}$ and the mass of the in-flight fluid mass for a single radiator from Eq. 11 is $M_{f}=3.31 \times 10^{4} \mathrm{~kg}$. We therefore note that the mass-specific heat rejection of the radiating surface is

$$
K W / \mathrm{kg}=\frac{1.243 \times 10^{6}}{3.31 \times 10^{4}}=37.56 \frac{\mathrm{MW}}{\mathrm{mT}}
$$

which is approximately 7.5 times more effective than the conventional radiator of $5 \mathrm{MW} / \mathrm{mT}$. The radiator mass for the case in Table 1 , namely $240 \mathrm{mT}$ now becomes with the liquid droplet design 6.39 $\mathrm{mT}$ which in turn makes the total vehicle mass $180 \mathrm{mT}$. With this mass the round trip to Mars will be 112 days instead of 169 days for the case with conventional radiator. No mirror magnets were needed on the premise that field reversal obviated the need for it, and the magnet mass was that of the central field extended over the length of the rocket.

\section{Conclusion}

We have examined in the paper two approaches to reducing the mass of the gasdynamic mirror fusion propulsion system. The first deals with the large magnetic fields at the mirrors which can be almost eliminated with the aid of field reversal that can be introduced in that region without hampering the propulsive capability of GDM. The other addresses the radiator problem by replacing the conventional radiator with the liquid droplet design that disposes of waste heat almost 8 times more efficiently. When these factors are taken into account it is seen that the total vehicle mass is reduced by over $50 \%$ resulting in a round trip journey to Mars in 112 days instead of 169 days. 


\section{Acknowledgment}

This work was supported by the U.S. National Aeronautics and Space Administration (NASA).

\section{$\underline{\text { References }}$}

1. T. Kammash and M. J. Lee, J. Propulsion and Power, 11, 544 (1995).

2. T. Kammash, M. J. Lee, and D. I. Poston, J. Propulsion and Power, 13, 421 (1997).

3. K. Miller, J. Slough, and A. Hoffman, AIP Conference Proceedings, 420, Part 3, p. 1352 (1998).

4. A. N. Nesmeyanov, Vapor Pressure of the Elements, Academic Press (1964).

5. A. T. Mattick and A. Hertzberg, Acta Astronautica, 12, 591 (1985).

6. R. T. Tausig and A. T. Mattick, J. Spacecraft and Rockets, 23, 10 (1986). 